\title{
Phase Transition Investigations of a Series of Aromatic Naphthalene-2-yl-4-(Alkoxy) Benzoate and Naphthalene-1-yl-4-(Alkoxy) Benzoate Materials
}

\author{
Wajdi Michael Zoghaib ${ }^{1 *}$, Carlo Carboni², Mohammed Elias Molla ${ }^{3}$, Samiya Al-Mahrezi², \\ Turkiya Al-Shahumi², Sanaa Al-Badi' ${ }^{1}$, Muna Al-Farsi ${ }^{1}$
}

${ }^{1}$ Department of Chemistry, Sultan Qaboos University, Al-Khod, Sultanate of Oman

${ }^{2}$ Department of Physics, Sultan Qaboos University, Al-Khod, Sultanate of Oman

${ }^{3}$ Department of Chemistry, Jahangirnagar University, Dhaka, Bangladesh

Email: ^zoghaibw@squ.edu.om

How to cite this paper: Zoghaib, W.M., Carboni, C., Molla, M.E., Al-Mahrezi, S., AlShahumi, T., Al-Badi, S. and Al-Farsi, M. (2022) Phase Transition Investigations of a Series of Aromatic Naphthalene-2-yl-4-(Alkoxy) Benzoate and Naphthalene-1-yl-4-(Alkoxy) Benzoate Materials. World Journal of Condensed Matter Physics, 12, 1-7. https://doi.org/10.4236/wjcmp.2022.121001

Received: January 6, 2022

Accepted: February 12, 2022

Published: February 15, 2022

Copyright $\odot 2022$ by author(s) and Scientific Research Publishing Inc. This work is licensed under the Creative Commons Attribution International License (CC BY 4.0).

http://creativecommons.org/licenses/by/4.0/

\begin{abstract}
The mesogenic properties of a homologous series of aromatic ester materials are investigated. Single tail and double tail materials were synthesized to compare with other series we prepared and published earlier. The phase sequences and transition temperatures were obtained by polarized light microscopy and differential scanning calorimetry. Only the single tail materials with the ester attached to naphthalene at position 2 display mesogenicbehavior. Two materials (ZH $29 \& \mathrm{ZH} \mathrm{32)}$ possess a monophasic nematic phase and another two materials (ZH $14 \&$ ZH 35) display biphasic nematic and smectic (A) phases.
\end{abstract}

\section{Keywords}

Liquid Crystals, Mesomorphism, Calamitic, Nematic, Smectic A, Polarized Optical Microscopy, Differential Scanning Calorimetry

\section{Introduction}

Liquid crystalline materials (LCs) possess a fourth phase where constituent molecules display a certain degree of fluid mobility to be classified as liquids in addition to having sufficient order to be called solid crystals. This unique combination of mobility and crystalline ordering distinguishes this particular phase of matter from the three very well-defined phases of matter (solid, liquid and gas) 
in that liquid crystalline materials have very useful applications in industry, academia and medicine. Among the few different classes of liquid crystals, thermotropic liquid crystals (where the phase is a function of temperature) in particular are of great technological importance in display devices and other industrial applications [1] [2] [3] [4] [5]. In many low molar mass calamiticmesogens (where molecular length is much larger than breadth), the rigid core is made up of a number of 1,4-disubstituted aromatic rings (with or without lateral substituents) terminated on one or both sides with a long flexible hydrocarbon chain head or tail (with or without halo substituents). The mesomorphic behavior of liquid crystal materials can be modified by altering their structure (head and tail length) and/or incorporating small size lateral substituents such as fluorine atoms in the rigid core [6]-[11].

There are three structural types of liquid crystals, all of which have their technological applications. The rod-like (calamitic) which was identified after the early discovery of liquid crystals by F. Reinitzer in 1888, the disc-like (discotic), discovered by S. Chandrasekhar in 1977 and finally the bowl-like (bowlic) proposed by L. Lam in 1982 [12]. The liquid crystalline materials discussed in this study are structurally closer to calamitic (rodic) where the longitudinal molecular axis is significantly larger than its width.

Thermotropic liquid crystals are mesogenic molecules in which the exhibited mesomorphism depends entirely on varying the surrounding temperature. In nematic-thermotropic LCs, a long-range orientational order exists whereby the longitudinal molecular axes align themselves parallel to a director axis $\mathrm{n}$. In smectic-thermotropic LCs, the aforementioned orientational order exists alongside a translational order whereby the molecules are arranged in equidistant planes forming a periodic layered structure possessing a certain layer thickness [1] [5] [13].

Three series of materials (A, B \& C) are investigated in this study. Series A contains a single long-chain hydrocarbon tail attached to a benzoic acid ester benzene ring via an ether link at benzene carbon number 4 . The benzoic acid tetrahedral oxygen is bonded to a naphthalene ring at position 2 forming the homologous series naphthalene-2-yl-4-(alkoxy) benzoate (Figure 1).

In the second series (B), the benzene ring with an ether-linked hydrocarbon tail at position 4 is bonded via an ester linkage to a naphthalene ring at position 1 forming the homologous series naphthalene-1-yl-4-(alkoxy) benzoate (Figure 2).

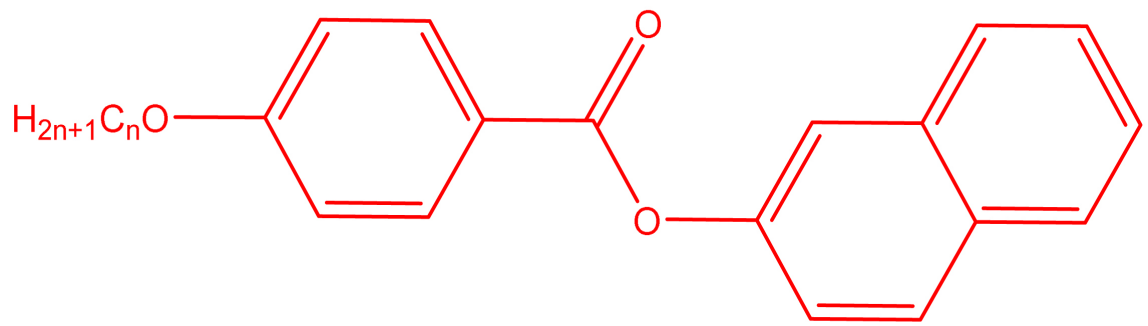

Figure 1. General structure of homologous series (A) members. 


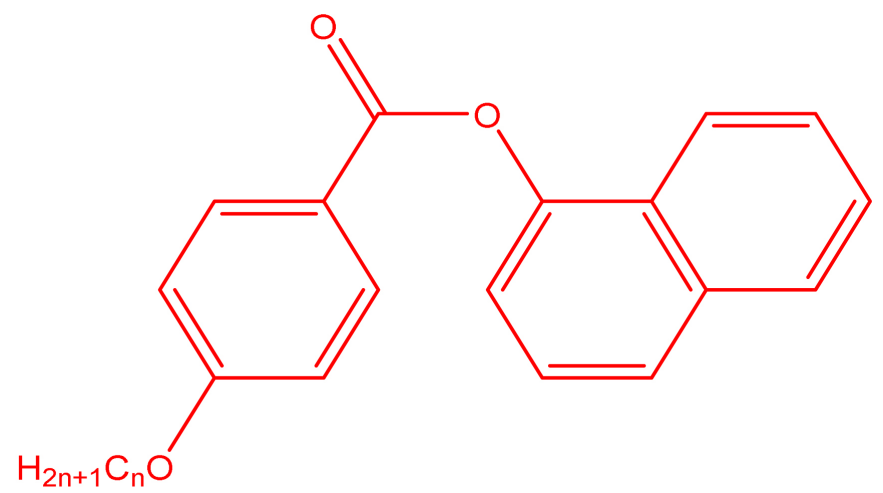

Figure 2. General structure of homologous series (B) members.

In the third series $(\mathrm{C})$, the benzoic acid benzene ring has two hydrocarbon chain tails ether-linked at positions 3 and 5. The benzoic acid tetrahedral oxygen is bonded to a naphthalene ring at position 2 forming the homologous series naphthalene-2-yl-3,5-bis(alkoxy) benzoate (Figure 3).

The three series in this study were synthesized in order to compare their mesogenic behavior or lack of it with previous materials we prepared, examined and published [14] [15]. The well-established and documented synthesis of these three series will be described elsewhere. This study describes the mesomorphic behavior of series (A) and the lack of it in series (B \& C), correlates tail chain length with mesogenic phases and temperatures and correlates structure with mesomorphic behavior or lack of it among the three series, A, B and C.

\section{Materials and Methods}

The materials' phase sequences were determined by polarized optical microscopy (POM) and differential scanning calorimetry (DSC). For polarized optical microscopy observations, an Olympus $\mathrm{BH} 2$ polarizing microscope equipped with a graduated rotation stage was used. The specimens were contained in $5 \mu \mathrm{m}$ gap commercial glass cells treated for planar alignment and coated on the inside with a thin layer of indium tin oxide (ITO). The specimen temperature was varied and controlled to $\pm 0.1^{\circ} \mathrm{C}$ using a heating/freezing stage temperature controller from Linkam (TMS 94).

The endothermic or exothermic energy with respect to temperature can be measured using DSC studies. Generally, when the LC complex is cooled, exothermic peaks are recorded; the area under the peaks along with the weight of the sample provides the energy possessed by the respective mesogenic phase. Similarly, when the liquid crystal is heated the endothermic peaks are observed. DSC measurements were acquired on a TA DSC Q20 model with a temperature range of $-40^{\circ} \mathrm{C}$ to $400^{\circ} \mathrm{C}$. A heating/cooling rate of $3.00^{\circ} \mathrm{C} / \mathrm{min}$ was used throughout the scanning procedure.

\section{Results}

The materials' molecular structures are provided in Figures 1-3 for series (A), 


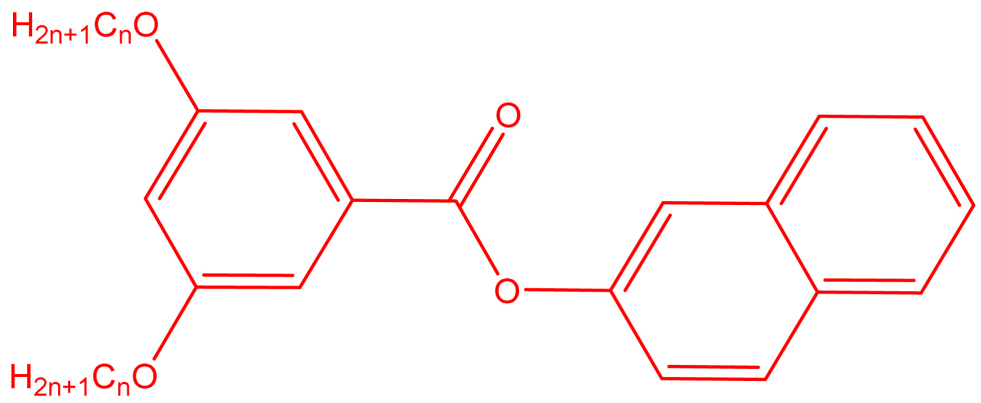

Figure 3. General structure of homologous series (C) members.

(B) and (C) respectively. The code names, POM transition temperatures (heating/cooling) as well as DSC heating/cooling data together are provided in tables 1, 2 and 3 for series (A), (B) and (C) respectively. A sample DSC thermogram for one of the samples is presented in Figure 4.

The transition temperatures observed by polarized optical microscopy agree within $\pm 2^{\circ} \mathrm{C}$ with the DSC recordings. Most materials display monotropic behavior, the mesophases are observed upon cooling and/or heating with two mesophases in some cases observed upon cooling in series (A). In the mesophases, the materials have a tendency to crystallize at a fixed temperature.

In series (A), Table 1, where the ether tailed benzene ring is linked to a naphthalene (at position 2) via an ester linkage, all materials in this series display a nematic phase upon cooling from the isotropic. The materials with the longest chains, ZH35 and ZH14 display on cooling a smectic A phase below the nematic phase. A double headed arrow for a transition indicates a reversible transition.

In series (B), Table 2, where the ether tailed benzene ring is linked via an ester linkage to the naphthalene position 1; upon heating all the materials melt from the crystal to the isotropic phase. Upon cooling, the materials do not display any mesophases.

In series (C), Table 3, where the dual ether tailed (positions 3 and 5) benzene ring is linked via an ester linkage to the naphthalene position 2; upon heating all the materials melt from the crystal to the isotropic phase. Upon cooling, the materials do not display any mesophases.

\section{Discussion}

It appears from the aforementioned observations that in series (A) materials with longer chain hydrocarbon ether tail, (12 and 10 carbons) two mesophases appear upon cooling namely nematic and smectic A. Whereas materials with shorter chain hydrocarbon ether tail ( 8 and 6 carbons) exhibit only a nematicmesophase.

In series (B) materials, no mesomorphism was observed either in long chain or short chain tail materials. Structurally speaking, and comparing the two materials in series (A) and (B) we notice that in series (A) molecules where the ester linkage is to naphthalene carbon \# 2, the molecules are closer to calamitic elongated (rod shaped) structures whereas in series (B), where the ester linkage is to 


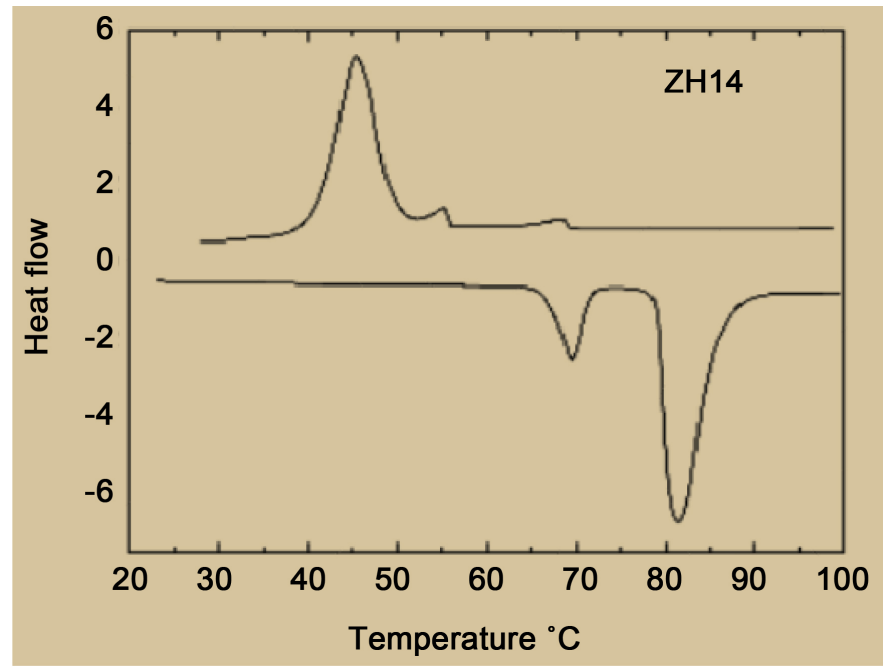

Figure 4. DSC thermogram of liquid crystal ZH 14 showing cooling mode (upper) and heating mode (lower).

Table 1. POM and DSC observations for series (A) members, temperatures are in ${ }^{\circ} \mathrm{C}$; the numbers in parenthesis indicate the transition enthalpy in units of $\mathrm{J} / \mathrm{g}$.

\begin{tabular}{|c|c|c|c|c|c|}
\hline \multirow{2}{*}{ Code } & \multirow{2}{*}{$\mathbf{n}$} & POM & POM & DSC & \multirow{2}{*}{$\begin{array}{c}\text { DSC } \\
\text { Heating }\end{array}$} \\
\hline & & Cooling & Heating & Cooling & \\
\hline \multirow{2}{*}{ ZH35 } & \multirow{2}{*}{12} & \multirow{2}{*}{$\stackrel{69.5}{\mathrm{I}} \stackrel{61}{\leftrightarrow} \mathrm{N} \stackrel{\leftrightarrow}{\leftrightarrow} \mathrm{S}_{\mathrm{A}} \stackrel{48}{\rightarrow} \mathrm{K}$} & \multirow{2}{*}{$\mathrm{K} \stackrel{62}{\rightarrow} \mathrm{N} \stackrel{71}{\leftrightarrow} \mathrm{I}$} & $\mathrm{I} \stackrel{71}{\leftrightarrow} \mathrm{N} \stackrel{62.5}{\leftrightarrow} \mathrm{S}_{\mathrm{A}} \stackrel{49.5}{\rightarrow} \mathrm{K}$ & $\mathrm{K} \stackrel{50}{\rightarrow} \mathrm{N} \stackrel{70}{\leftrightarrow} \mathrm{I}$ \\
\hline & & & & $(1.20) \quad(1.52) \quad(77.8)$ & $(91.4) \quad(1.34)$ \\
\hline \multirow{2}{*}{ ZH14 } & \multirow{2}{*}{10} & \multirow[t]{2}{*}{$\stackrel{68.5}{\mathrm{I}} \stackrel{55}{\leftrightarrow} \mathrm{N} \stackrel{\leftrightarrow}{\leftrightarrow} \mathrm{S}_{\mathrm{A}} \stackrel{46}{\rightarrow} \mathrm{K}$} & \multirow{2}{*}{$\mathrm{K} \stackrel{56}{\rightarrow} \mathrm{N} \stackrel{69}{\leftrightarrow} \mathrm{I}$} & $\mathrm{I} \stackrel{69}{\leftrightarrow} \mathrm{N} \stackrel{56}{\leftrightarrow} \mathrm{S}_{\mathrm{A}} \stackrel{47}{\rightarrow} \mathrm{K}$ & $\mathrm{K} \stackrel{48}{\rightarrow} \mathrm{N} \stackrel{70}{\leftrightarrow} \mathrm{I}$ \\
\hline & & & & $(1.52) \quad(1.18) \quad(29.2)$ & $(86.9) \quad(1.28)$ \\
\hline \multirow{2}{*}{ ZH32 } & \multirow{2}{*}{8} & \multirow{2}{*}{$\mathrm{I} \stackrel{65}{\leftrightarrow} \mathrm{N} \stackrel{55}{\rightarrow} \mathrm{K}$} & \multirow{2}{*}{$\mathrm{K} \rightarrow \mathrm{I3.5}$} & 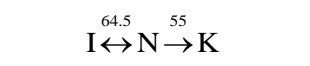 & \multirow{2}{*}{$\begin{array}{c}\mathrm{K} \stackrel{64}{\rightarrow} \mathrm{I} \\
(60.76)\end{array}$} \\
\hline & & & & $(0.85) \quad(23.60)$ & \\
\hline \multirow{2}{*}{$\mathrm{ZH} 29$} & \multirow{2}{*}{6} & \multirow{2}{*}{$\mathrm{I} \stackrel{63}{\leftrightarrow} \mathrm{N} \stackrel{50}{\rightarrow} \mathrm{K}$} & \multirow{2}{*}{$\mathrm{K} \stackrel{51}{\rightarrow} \mathrm{N} \stackrel{62.5}{\leftrightarrow} \mathrm{I}$} & $\mathrm{I} \stackrel{62}{\leftrightarrow} \mathrm{N} \stackrel{50}{\rightarrow} \mathrm{K}$ & $\mathrm{K} \rightarrow \mathrm{51.5} \rightarrow \mathrm{N} \stackrel{63}{\leftrightarrow} \mathrm{I}$ \\
\hline & & & & (1.15) (61.84) & $(70.16) \quad(1.12)$ \\
\hline
\end{tabular}

Table 2. POM observations for series (B) members, temperatures are in ${ }^{\circ} \mathrm{C}$. No mesomorphism observed in this series.

\begin{tabular}{|c|c|c|c|}
\hline Code & $\mathbf{n}$ & POM (Heating) & POM (cooling) \\
\hline $\mathrm{ZH} 34$ & 12 & $\mathrm{~K} \stackrel{79}{\rightarrow} \mathrm{I}$ & $\mathrm{I} \stackrel{73}{\rightarrow} \mathrm{K}$ \\
\hline $\mathrm{ZH} 13$ & 10 & $\mathrm{~K} \stackrel{70}{\rightarrow} \mathrm{I}$ & $\mathrm{I} \stackrel{65}{\rightarrow} \mathrm{K}$ \\
\hline ZH31 & 8 & $\mathrm{~K} \stackrel{68}{\rightarrow} \mathrm{I}$ & $\mathrm{I} \stackrel{62}{\rightarrow} \mathrm{K}$ \\
\hline $\mathrm{ZH} 28$ & 6 & $\mathrm{~K} \stackrel{62}{\rightarrow} \mathrm{I}$ & $\mathrm{I} \stackrel{55}{\rightarrow} \mathrm{K}$ \\
\hline
\end{tabular}

Table 3. POM observations for series (C) members, temperatures are in ${ }^{\circ} \mathrm{C}$. No mesomorphism observed in this series.

\begin{tabular}{cccc}
\hline Code & $\mathrm{n}$ & POM (Heating) & POM (cooling) \\
\hline ZH20 & 8 & $\mathrm{~K} \stackrel{61}{\rightarrow} \mathrm{I}$ & $\mathrm{I} \rightarrow \mathrm{K}$ \\
ZH16 & 6 & $\mathrm{~K} \stackrel{76}{\rightarrow} \mathrm{I}$ & $\mathrm{I} \rightarrow \mathrm{K}$ \\
\hline
\end{tabular}


naphthalene carbon \# 1, the general molecular structure is bent and deviates significantly from rod-shaped calamitic geometry.

In series (C) materials, we expected to observe some mesophase behavior however there was none. Our expectations were based on the fact that the ester linkage in series (C) is the same as that in series (A). However, it appears that the presence of two hydrocarbon chains ether linked to the benzoic acid ring at positions 3 and 5 in series (C) distorts the overall molecular geometry away from a rod-shaped calamitic structure.

\section{Conclusions}

Three series of calamitic materials were prepared via established synthetic methods to compare their mesomorphic behavior or lack of mesomorphism with materials we prepared, examined and published earlier. Only one of the three series examined in this study displays mesophases and thus series (A) members are liquid crystalline materials. ZH 14 \& ZH 35 display biphasic nematic as well as smectic (A) phases, whereas ZH 29 \& ZH 32 possess a monophasic nematic phase. We conclude that in series (A), since the general molecular structure is more rod-like (calamitic) than in the other two series, mesomorphism is displayed.

We also conclude that in series (A), longer ether chain tails display two mesomorphic phases namely smectic $A$ and nematic whereas shorter ether chain tails exhibit only a nematic phase.

\section{Acknowledgements}

The authors are grateful to Sultan Qaboos University and Jahangirnagar University for providing facilities and financial support to make this research possible.

\section{Conflicts of Interest}

The authors declare no conflicts of interest regarding the publication of this paper.

\section{References}

[1] Collings, P.J. and Hird, M. (1998) Introduction to Liquid Crystals: Chemistry and Physics. CRC Press, London. https://doi.org/10.4324/9780203211199

[2] Bahadur, B. (1992) Liquid Crystals: Applications and Uses. Vol. 3, World Scientific, Singapore, 68-211. https://doi.org/10.1142/1300

[3] Kaafarani, B.R. (2011) Discotic Liquid Crystals for Opto-Electronic Applications. Chemistry of Materials, 23, 378-396. https://doi.org/10.1021/cm102117c

[4] Sluckin, T.J., Dunmur, D.A. and Stegemeyer, H. (2004) Crystals That Flow. $1^{\text {st }}$ Edition, CRC Press, Boca Raton. https://doi.org/10.1201/9780203022658

[5] Lin, Y.H., Wang, Y.J. and Reshetnyak, V. (2017) Liquid Crystal Lenses with Tunable Focal Length. Liquid Crystals Reviews, 5, 111-143. https://doi.org/10.1080/21680396.2018.1440256

[6] Gray, G.W. (1962) Molecular Structure and the Properties of Liquid Crystals. Academic Press, London and New York. 
[7] Demus, D., Goodby, J., Gray, G.W., Spiess, H.W. and Vill, V. (2011) Handbook of Liquid Crystals: Low Molecular Weight Liquid Crystals I: Calamitic Liquid Crystals. Wiley, London.

[8] Zoghaib, W.M., Carboni, C., George, A.K., AL-Manthari, S., Al-Hussaini, A. and AlFutaisi, F. (2011) Novel Fluorinated Ferroelectric Organosiloxane Liquid Crystals. Molecular Crystals and Liquid Crystals, 542, 123-131. https://doi.org/10.1080/15421406.2011.570148

[9] Zoghaib, W.M., Carboni, C., Al-Hinai, H., Al-Abri, S., Al-Kasbi, S., Al-Nasseri, E., Al-Masroori, M., Al-Yahyaee, M. and Al-Busaidi, S. (2015) A Novel Series of Chiral Fluorinated Organosiloxane Liquid Crystals. Molecular Crystals and Liquid Crystals, 612, 183-190. https://doi.org/10.1080/15421406.2015.1030984

[10] Zoghaib, W.M., Carboni, C., Al-Rawahi, J., Al-Rubaiei, F., Al-Bulushi, H., Al-Aufi, M., Al-Harrasi, M., Al-Kalbani, S. and Al-Kiyumi, A. (2016) Bifurcated-Tail Chiral Fluorinated Organosiloxane Liquid Crystalline Materials. Molecular Crystals and Liquid Crystals, 632, 114-123.

[11] Zoghaib, W.M., Carboni, C., Kashoub, F.A., Al-Rushidi, J.S., Al-Jabri, B.Y., Al-Alawi, A.S. and Al-Mendhry, H.F. (2018) A de Vries (SmC*) Phase in a Novel Series of Chiral Fluorinated Organosiloxane Liquid Crystals. Molecular Crystals and Liquid Crystals, 666, 54-64. https://doi.org/10.1080/15421406.2018.1549158

[12] Wang, L., Huang, D., Lam, L. and Cheng, Z. (2017) Bowlics: History, Advances and Applications. Liquid Crystals Today, 26, 85-111. https://doi.org/10.1080/1358314X.2017.1398307

[13] Lagerwall, J.P.F. and Giesselmann, F. (2006) Current Topics in Smectic Liquid Crystal Research. ChemPhysChem, 7, 20-45. https://doi.org/10.1002/cphc.200500472

[14] Zoghaib, W.M., George, A.K., Carboni, C., Molla, M.E., Al-Mahrezi, S., Al-Khan, H. and Al-Kamshaki, D. (2020) Mesomorphism of a Series of Aromatic Single Tail Biphenyl-yl-4-(alkoxy) Benzoate and 4-(Benzyloxy)phenyl-4-(alkoxy)benzoate Liquid Crystalline Materials. Molecular Crystals and Liquid Crystals, 710, 13-18. https://doi.org/10.1080/15421406.2020.1824171

[15] Al-Yazeedi, R. (2015) A Phase Transition Study on Liquid Crystalline Materials Using Polarized Optical Microscopy and Differential Scanning Calorimetry. Final Year Project Thesis Submitted to the Chemistry Department, Sultan Qaboos University, Oman (Unpublished Results). 